\title{
SUMS OF BIQUADRATES AND ELLIPTIC CURVES
}

\author{
Julián Aguirre and Juan Carlos Peral \\ University of the Basque Country, UPV/EHU, Spain
}

\begin{abstract}
Given the family of elliptic curves $y^{2}=x^{3}-\left(1+u^{4}\right) x$, $u \in \mathbb{Q}$, or equivalently $y^{2}=x^{3}-\left(m^{4}+n^{4}\right) x$ for $m, n$ integers, we prove that its rank over $\mathbb{Q}(u)$ is 2 . We also show the existence of subfamilies of rank at least 3 and 4 over $\mathbb{Q}(u)$. Also, assuming the Parity Conjecture, we prove the existence of infinitely many curves having rank at least 5 over $\mathbb{Q}$.

Performing an exhaustive search in the range $1 \leq n<m \leq 251000$ we have found more than 1500 curves with rank 8, over 150 with rank 9, nine of rank 10 and one of rank 11. This improves previous results of Izadi, Khoshnam and Nabardi.
\end{abstract}

\section{INTRODUCTION}

Curves of the form $y^{2}=x^{3}-B x$ have $j$-invariant equal to 1728 and complex multiplication. They have been studied by many authors, see for example $[1,8,9,13,14,17,19,21]$ and the references therein. An example with rank 14 was found by Watkins within the family constructed in [1].

Several families of integers $B$ 's have been considered. For instance, the case $B=d^{2}$ is known as the congruent number problem and it corresponds to the quadratic twist by $d$ of the elliptic curve $y^{2}=x^{3}-x$. For the current rank records in every possible torsion group see [7].

In this paper we consider the family of curves

$$
y^{2}=x^{3}-\left(m^{4}+n^{4}\right) x, \quad m, n \in \mathbb{N} .
$$

There is no loss of generality in assuming $m^{4}+n^{4}$ to be fourth power free, so we will assume from now on that $\operatorname{gcd}(m, n)=1$. The change of variables

2010 Mathematics Subject Classification. 11G05.

Key words and phrases. Elliptic curve, rank, biquadrate.

J. Aguirre supported by grant IT-305-07 of the Basque Government. J.C. Peral supported by the UPV/EHU grant EHU 10/05. 
$y / m^{3} \rightarrow y, x / m^{2} \rightarrow x, u=n / m$ shows that $\mathcal{E}_{m, n}$ is isomorphic to the oneparameter family

$$
y^{2}=x^{3}-\left(1+u^{4}\right) x, \quad u \in \mathbb{Q} .
$$

We prove that $\mathcal{E}_{u}$ has rank 2 over $\mathbb{Q}(u)$. By imposing the existence of a third independent point, or equivalently by properly choosing $u$, we show the existence of a subfamily with rank at least 3 .

Euler and Steggall constructed parametric families of integers expressible in two different ways as a sum of two biquadrates. Using these parameterizations we prove that there exist two subfamilies with rank at least 4 over $\mathbb{Q}(u)$. Moreover all the curves of the second family have root number -1 , and therefore, assuming the Parity Conjecture, all but a finite number have rank at least 5 over $\mathbb{Q}$.

We finish by giving the results of a massive search for curves of high rank. The computations were done using Mathematica ${ }^{\circledR}[15]$ and Cremona's mwrank [5].

This is an expanded version of [2] by the same authors.

REMARK 1.1. In [9], which appeared simultaneously with [2], (March 2012), the same family of elliptic curves is considered. The authors study several families of curves, included one studied here. They prove the existence of subfamilies having rank at least 3 and 4 over $\mathbb{Q}(u)$, and they also use the Euler parameterization in order to prove the existence of rank 4 families. They do not show the existence of infinitely many curves of rank at least 5 over $\mathbb{Q}$ and they show particular examples of curves having rank 7 and 8 . In both respects our paper represents an improvement of their results.

1.1. The root number of $\mathcal{E}_{m, n}$. Since $m$ and $n$ are relatively prime, if $p \neq 2$ is a prime divisor of $m^{4}+n^{4}$, then -1 is a quadratic residue modulo $p$ and $p \equiv 1(\bmod 4)$.

If $B \not \equiv 0(\bmod 4)$, then the root number of the curve $y^{2}=x^{3}-B x$ is (see $[18])$

$$
\omega(B)=\operatorname{sign}(-B) \epsilon(B) \prod_{p^{2} \| B, p \geq 3}\left(\frac{-1}{p}\right),
$$

where

$$
\epsilon(B)=\left\{\begin{aligned}
-1 & \text { if } B \equiv 1,3,11,13 \quad(\bmod 16) \\
1 & \text { if } B \equiv 2,5,6,7,9,10,14,15 \quad(\bmod 16) .
\end{aligned}\right.
$$

As observed before, all odd prime divisors of $m^{4}+n^{4}$ are $\equiv 1(\bmod 4)$, so that the product of the Legendre symbols in the above formula is equal to 1 . On the other hand, fourth powers are 1 or 0 modulo 16 and $\operatorname{sign}\left(m^{4}+n^{4}\right)=1$, so that the root number of $\mathcal{E}_{m, n}$ is

$$
\omega\left(\mathcal{E}_{m, n}\right)=\left\{\begin{aligned}
1 & \text { if } m^{4}+n^{4} \text { odd } \\
-1 & \text { if } m^{4}+n^{4} \text { even }
\end{aligned}\right.
$$


1.2. The torsion group of $\mathcal{E}_{m, n}$. The torsion group of the elliptic curve $y^{2}=x^{3}-B x$ is $\mathbb{Z} / 4 \mathbb{Z}$ if $B=-4, \mathbb{Z} / 2 \mathbb{Z} \times \mathbb{Z} / 2 \mathbb{Z}$ if $B$ is a perfect square, and $\mathbb{Z} / 2 \mathbb{Z}$ in the rest of the cases (see [22, Proposition 6.1]). Since $m^{4}+n^{4}$ is never a perfect square (see [11, Theorem 266]), the torsion group of $\mathcal{E}_{m, n}$ is $\mathbb{Z} / 2 \mathbb{Z}$.

1.3. Notation. Given an elliptic curve $\mathcal{E}$ over a field $\mathbb{K}$, we define $\operatorname{rank}(\mathcal{E}(\mathbb{K}))$ as the rank of $\mathcal{E}$ over $\mathbb{K}$; if no field is mentioned, it is understood that the field is $\mathbb{Q}$. If $\mathcal{E}$ is an elliptic curve over $\mathbb{Q}, \operatorname{Selmer}_{2}(\mathcal{E})$ denotes its 2-Selmer rank.

\section{General CASE: RANKS 2 AND 3}

In this section we prove the main result about the rank of the family $\mathcal{E}_{u}$.

TheOREM 2.1. a) The rank of $\mathcal{E}_{u}$ over $\mathbb{Q}(u)$ is 2 .

b) There exists a subfamily of $\mathcal{E}_{u}$ whose rank is at least 3 over $\mathbb{Q}(u)$.

Proof. a) We find that the following two points are on the curve:

$$
P_{1}(u)=\left(-u^{2}, u\right), \quad P_{2}(u)=\left(-1, u^{2}\right) .
$$

In order to prove that the family $\mathcal{E}_{u}$ has rank at least 2 over $\mathbb{Q}(u)$, it suffices to find a specialization $u=u_{0}$ such that the points $P_{1}\left(u_{0}\right)$ and $P_{2}\left(u_{0}\right)$ are independent points on the specialized curve over $\mathbb{Q}$, since the specialization is an injective homomorphism for all but finitely many values of the parameter (see $\left[22\right.$, Theorem 11.4, pp. 271].) For $u_{0}=2$ the curve is $y^{2}=x^{3}-17 x$ and the points are

$$
P_{1}(2)=(-4,2), \quad P_{2}(2)=(-1,4) .
$$

These two points turn out to be independent, as shown by mwrank, and therefore the rank of the family $\mathcal{E}_{u}$ is at least 2 over $\mathbb{Q}(u)$.

Next we show that the rank of $\mathcal{E}_{u}$ over $\mathbb{Q}(u)$ is exactly equal to 2 . We get an upper bound for $\operatorname{rank}\left(\mathcal{E}_{u}(\mathbb{C}(u))\right)$ using Shioda's formula ([20, Corollary $5.3])$ :

$$
\operatorname{rank}\left(\mathcal{E}_{u}(\mathbb{C}(u))\right)=\operatorname{rank} N S\left(\mathcal{E}_{u}, \mathbb{C}\right)-2-\sum_{s}\left(m_{s}-1\right) .
$$

Here $N S\left(\mathcal{E}_{u}, \mathbb{C}\right)$ is the Néron-Severi group of $\mathcal{E}_{u}$ over $\mathbb{C}$, and the sum ranges over all singular fibers of $\mathcal{E}_{u}$, with $m_{s}$ being the number of irreducible components of the fiber. Since $\mathcal{E}_{u}$ has short Weierstrass form $y^{2}=x^{3}+$ $C(u) x+D$ with $C(u)$ a polynomial of degree 4 in $u$ and $D=0, \mathcal{E}_{u}$ is a rational surface (see $[3,16]$ ), and hence $\operatorname{rank} N S\left(\mathcal{E}_{u}, \mathbb{C}\right)=10$. The numbers $m_{s}$ can be determined from Kodaira fibers (see [16, Section 4]), which are given by the four roots of the discriminant $\Delta=4\left(1+u^{4}\right)^{3}$, all of them of type III. Therefore

$$
\operatorname{rank}\left(\mathcal{E}_{u}(\mathbb{C}(u))\right) \leq 10-2-1-1-1-1=4 .
$$


Thus we now know that $2 \leq \operatorname{rank}\left(\mathcal{E}_{u}(\mathbb{Q}(u))\right) \leq 4$. Let us consider now the curve $\mathcal{E}_{u}$ over $\mathbb{K}(u)$, where $\mathbb{K}=\mathbb{Q}(\sqrt{-1})$. Since $\mathcal{E}_{u}$ is isomorphic to its $(-1)$-twist, its rank over $\mathbb{K}(u)$ is at least 4 and the preceding inequality is an equality. It follows that the rank over $\mathbb{Q}(u)$ is 2 .

b) Now we force the existence of a new point on $\mathcal{E}_{u}$ by choosing a subfamily of parameters, and we prove the independence of the new point with respect to $P_{1}$ and $P_{2}$. We impose $-u^{2}+1$ as the $x$-coordinate of a new point on $\mathcal{E}_{u}$. This is equivalent to $2\left(u^{2}-1\right)$ being a rational square or to choose $u=\left(w^{2}+2\right) /\left(w^{2}-2\right)$. Once we clear denominators and rename the parameter by $u$ we have the new family given by

$$
y^{2}=x^{3}-2\left(16+24 u^{4}+u^{8}\right) x .
$$

The three points are

$$
\begin{aligned}
& P_{1}(u)=\left(-\left(2+u^{2}\right)^{2},\left(-2+u^{2}\right)^{2}\left(2+u^{2}\right)\right), \\
& P_{2}(u)=\left(-\left(-2+u^{2}\right)^{2},\left(-2+u^{2}\right)\left(2+u^{2}\right)^{2}\right), \\
& P_{3}(u)=\left(-8 u^{2}, 4 u\left(-2+u^{2}\right)\left(2+u^{2}\right)\right) .
\end{aligned}
$$

For $u=1$ the curve is $y^{2}=x^{3}-82 x$ and the specialized points are

$$
P_{1}(1)=(-9,3), \quad P_{2}(1)=(-1,-9) \quad \text { and } \quad P_{3}(1)=(-8,-12) .
$$

A calculation with mwrank shows that three points are independent. As before, a specialization argument implies that the rank of $(2.1)$ over $\mathbb{Q}(u)$ is at least 3.

REMARK 2.2. Other families of rank at least 3 can be found by imposing $u^{2}+1$ or $3 u^{2}-4 u+3$ as the $x$ coordinate of a new point. The first case is equivalent to choose $u=\left(-2+w^{2}\right) /\left(2-4 w+w^{2}\right)$, and the second to choose $u=\left(-6+w^{2}\right) /\left(-2+4 w+w^{2}\right)$.

\section{TWO REPRESEnTATIONS AS SUM OF BIQUADRATES: RANKS 4 AND 5}

In this section we give results about the rank of curves $y^{2}=x^{3}-B x$ when $B$ is the sum of two biquadrates in two different ways.

The diophantine equation

$$
m_{1}^{4}+n_{1}^{4}=m_{2}^{4}+n_{2}^{4}
$$

has been studied by many authors (see [6, pp.644-648] and [25]). Several parametric solutions are known. The list of solutions with

$$
\max \left(m_{1}, n_{1}, m_{2}, n_{2}\right) \leq 10^{7}
$$

can be found on the web page [24].

We will consider two parametric families of solutions of (3.1), the first one constructed by Euler and the second by J. Steggall. 
3.1. Euler's parameterization. Euler constructed a two parameter family of solutions of (3.1) homogeneous of degree 7 which can be written as

$$
m_{1}=f(u, v), \quad n_{1}=f(v,-u), \quad m_{2}=f(u,-v), \quad n_{2}=f(v, u),
$$

where

$$
f(u, v)=v\left(u^{6}+3 u^{5} v-2 u^{4} v^{2}+u^{2} v^{4}+v^{6}\right) .
$$

Because of the homogeneity, we may assume without loss of generality that $v=1$. This gives the family of solutions

$$
\begin{aligned}
m_{1}(u) & =1+u^{2}-2 u^{4}+3 u^{5}+u^{6}, \\
n_{1}(u) & =u\left(1-3 u-2 u^{2}+u^{4}+u^{6}\right), \\
m_{2}(u) & =1+u^{2}-2 u^{4}-3 u^{5}+u^{6}, \\
n_{2}(u) & =u\left(1+3 u-2 u^{2}+u^{4}+u^{6}\right) .
\end{aligned}
$$

We study the family of curves

$$
y^{2}=x^{3}-F(u) x, \quad F(u)=\left(m_{i}(u)\right)^{4}+\left(n_{i}(u)\right)^{4}, \quad i=1,2
$$

where $m_{i}(u), n_{i}(u), i=1,2$, are given by (3.2). Section 2 and the fact that $F(u)$ can be expressed in two different ways as sum of two biquadrates suggest that the subfamily $\mathcal{F}_{u}$ has rank greater than the rank of the general family $\mathcal{E}_{u}$, and that this generic rank is at least 4 . Moreover, the root number is 1 for all members of the family, so that if the Parity Conjecture is true, then the rank is even for al curves in the family.

In [12] the authors prove that if $F(u)$ is square free, then the rank of $\mathcal{F}_{u}$ over $\mathbb{Q}(u)$ is at least 3 . They also prove, assuming the Parity Conjecture and some other conditions, that the rank is at least 4 . We improve their result by proving in the next theorem that the rank is at least 4 over $\mathbb{Q}(u)$ unconditionally.

THEOREM 3.1. The rank of $\mathcal{F}_{u}$ over $\mathbb{Q}(u)$ is at least 4.

Proof. The four points

$$
\left(-\left(m_{i}(u)\right)^{2}, m_{i}(u)\left(n_{i}(u)\right)^{2}\right), \quad\left(-\left(n_{i}(u)\right)^{2}, n_{i}(u)\left(m_{i}(u)\right)^{2}\right), \quad i=1,2,
$$

are on the curve $\mathcal{F}_{u}$. Choose $u=2$. Then $\mathcal{F}_{u}$ becomes $y^{2}=x^{3}-$ $635318657 x$, whose rank is 4 , and the four points are $(-17689,2388148)$, $(-17956,2370326),(-3481,-1472876)$, and $(-24964,549998)$. A calculation with mwrank shows that they are independent. The same specialization argument as in Theorem 2.1 implies that the rank of $\mathcal{F}_{u}$ over $\mathbb{Q}(u)$ is at least 4 .

Remark 3.2. In [9, Section 3.3], the authors consider the same family and the same four points. They prove in Theorem 3.7 that the family has rank at least 4 over $\mathbb{Q}(u)$ by showing directly the independence of the four points. 
3.2. Steggall's parameterization. The following parameterization of (3.1) can be found in [23]:

$$
\begin{aligned}
m_{1}(u)= & -1+u+u^{2}+5 u^{3}+6 u^{4}-12 u^{5}-4 u^{6}+7 u^{7}-3 u^{8} \\
& -3 u^{9}+4 u^{10}+2 u^{11}-u^{12}+u^{13} \\
n_{1}(u)=1 & +u+2 u^{2}-4 u^{3}-3 u^{4}+3 u^{5}+7 u^{6}+4 u^{7}-12 u^{8} \\
& -6 u^{9}+5 u^{10}-u^{11}+u^{12}+u^{13}, \\
m_{2}(u)= & -1+u-2 u^{2}-4 u^{3}+3 u^{4}+3 u^{5}-7 u^{6}+4 u^{7}+12 u^{8} \\
& -6 u^{9}-5 u^{10}-u^{11}-u^{12}+u^{13}, \\
n_{2}(u)=1 & +u-u^{2}+5 u^{3}-6 u^{4}-12 u^{5}+4 u^{6}+7 u^{7}+3 u^{8} \\
& -3 u^{9}-4 u^{10}+2 u^{11}+u^{12}+u^{13} .
\end{aligned}
$$

As before, we consider the family

$$
y^{2}=x^{3}-S(u) x, \quad S(u)=m_{i}(u)^{4}+n_{i}(u)^{4}, \quad i=1,2,
$$

where $m_{i}(u)$ and $n_{i}(u), i=1,2$, are given by (3.3). The explicit formula of $S$ is

$$
\begin{aligned}
S(u)= & \left(2+4 u^{2}-10 u^{4}-24 u^{6}+57 u^{8}-24 u^{10}-10 u^{12}+4 u^{14}+2 u^{16}\right) \\
& \times\left(1+2 u^{2}-5 u^{4}-12 u^{6}+69 u^{8}-12 u^{10}-5 u^{12}+2 u^{14}+u^{16}\right) \\
& \times\left(1+4 u^{2}-u^{4}-24 u^{6}+41 u^{8}-24 u^{10}-u^{12}+4 u^{14}+u^{16}\right) \\
& \times\left(1+u^{4}\right) .
\end{aligned}
$$

Theorem 3.3. Assuming the Parity Conjecture all but a finite number of the curves of the family $\mathcal{S}_{u}$ have rank at least 5 over $\mathbb{Q}$.

Proof. The same proof as in the case of Euler's parameterization gives that the rank of $\mathcal{S}_{u}$ is at least 4 over $\mathbb{Q}(u)$ unconditionally. Since $S(u)$ is even when $u$ is an integer, the root number of $\mathcal{S}_{u}$ is -1 (see subsection 1.1 of the Introduction.) According to the Parity Conjecture all curves have odd rank and all but a finite number of them have rank $\geq 4$, and so should have rank at least 5 .

REMARK 3.4. The constructions given above suggest that if we had families of integers with many different representations as sum of two fourth powers, then it would be possible to construct families of elliptic curves, and examples of elliptic curves, having large ranks. Unfortunately, not even a single example with three different representations is known, see [10]. Moreover, a simple heuristic density argument tell us that the possibility of finding multiple representations of that form it is very unlikely. 


\section{SEARCH FOR CURVES OF High RANK AND RESUlts}

We have searched for curves of high rank (high meaning greater or equal to 8 ) in the family $\mathcal{E}_{m, n}$. It is tempting to carry out the search in the families $\mathcal{F}_{u}$ and $\mathcal{S}_{u}$, but the coefficients $F(u)$ and $S(u)$ are very large even for small values of the parameter $u$, and the computations soon become unfeasible. However, we have used mwrank on the 11089 curves coming from the solutions of (3.1) in [24]. We found 9 curves of rank 8, one of rank 9 and another one of rank 10.

We have also searched among the curves $\mathcal{E}_{m, n}$ with

$$
1 \leq m<n \leq 251000, \quad \operatorname{gcd}(m, n)=1 .
$$

To narrow the search, we have used two criteria to select values of $m$ and $n$ which are good candidates to produce curves $\mathcal{E}_{m, n}$ of high rank: one is the 2 Selmer rank and the other is based on Jacobi sums. Let $p_{k}^{*}$ be the $k$-th prime number congruent to one modulo four $\left(p_{1}^{*}=5, p_{2}^{*}=13, \ldots\right)$ and define for $B \in \mathbb{Z}$

$$
\sigma(N, B)=\sum_{k=1}^{N} \sum_{x=1}^{p_{k}^{*}-1}\left(\frac{x^{3}-B x}{p_{k}^{*}}\right) .
$$

These sums are related to the Mestre-Nagao sums. Large values of $\sigma(N, B)$ for several values of $N$ is an heuristic indication that the curve $y^{2}=x^{3}-B x$ has high rank (see [4].) The reason to consider only primes congruent to one modulo four is that when $B$ is the sum of two biquadrates, the inner sum for primes congruent to three modulo four is equal to zero because the polynomial $x^{3}-B x$ is odd.

For $2 \leq n \leq 50000$ we selected $m$ 's and $n$ 's such that

$$
\operatorname{Selmer}_{2}\left(\mathcal{E}_{m, n}\right) \geq 8, \quad \sigma\left(40, m^{4}+n^{4}\right) \geq 190 \quad \text { and } \quad \sigma\left(60, m^{4}+n^{4}\right) \geq 235 .
$$

The numerical values were chosen so that all the examples known before the search met the criteria. The search produced 1381 curves of rank 8 , 77 of rank 9 and one of rank 10. For $50000<n \leq 251000$ we focused on curves of rank at least 10 . To this end, we used the more restrictive criteria: $\operatorname{Selmer}_{2}\left(\mathcal{E}_{m, n}\right) \geq 10$ and

$\sigma\left(50, m^{4}+n^{4}\right) \geq 500, \quad \sigma\left(75, m^{4}+n^{4}\right) \geq 800 \quad$ and $\quad \sigma\left(100, m^{4}+n^{4}\right) \geq 1200$.

The numerical values 500, 800 and 1200 were chosen a little below of the median of $\sigma\left(50, m^{4}+n^{4}\right), \sigma\left(75, m^{4}+n^{4}\right)$ and $\sigma\left(100, m^{4}+n^{4}\right)$ for all the pairs $(m, n)$ found in the previous search with $\operatorname{rank}\left(\mathcal{E}_{m, n}\right) \geq 8$ respectively.

All searches combined found

- 1618 curves of rank 8 ,

- 169 curves of rank 9 ,

- 9 curves of rank 10 ,

- 1 curve of rank 11 . 
TABLE 1. Smallest $m^{4}+n^{4}$ providing a curve of the specified rank

\begin{tabular}{rrrrr}
\hline Rank & $m^{4}+n^{4}$ & $m$ & $n$ \\
\hline 2 & 17 & $\bullet$ & 1 & 2 \\
3 & 82 & $\bullet$ & 1 & 3 \\
4 & 6497 & $\bullet$ & 7 & 8 \\
5 & 520946 & & 19 & 25 \\
6 & 6096577 & $\bullet$ & 24 & 49 \\
7 & 3534242722 & 83 & 243 \\
8 & 25792915457 & $\bullet$ & 326 & 347 \\
9 & 228746044559762 & 2387 & 3743 \\
10 & 6101085725427704897 & 24523 & 48946 \\
11 & 3036966236612302373186 & 223985 & 151009 \\
\hline
\end{tabular}

TABLE 2. Curves $\mathcal{E}_{m, n}$ of rank $9,1 \leq m<n<12000$

\begin{tabular}{rrr}
\hline$m^{4}+n^{4}$ & $m$ & $n$ \\
\hline 228746044559762 & 2387 & 3743 \\
1575192445914242 & 973 & 6299 \\
3302246465649442 & 3837 & 7453 \\
7138308052996402 & 7267 & 8121 \\
9526110394254562 & 5511 & 9631 \\
10923258252017362 & 3047 & 10203 \\
12715483600163122 & 1757 & 10617 \\
13309386362157266 & 1745 & 10739 \\
13914233779769842 & 8817 & 9419 \\
14179340856132482 & 6593 & 10529 \\
15597163165713362 & 3961 & 11131 \\
\hline
\end{tabular}

Table 1 gives the smallest value of $m^{4}+n^{4}$ found for which $\operatorname{rank}\left(\mathcal{E}_{m, n}\right)=r$, $2 \leq r \leq 11$; a bullet means that it is in fact the smallest possible value. Table 2 contains the values of $m^{4}+n^{4}$ such that $1 \leq m<n<12000$ and $\operatorname{rank}\left(\mathcal{E}_{m, n}\right)=9$. Finally, the values of $m^{4}+n^{4} \operatorname{such}$ that $\operatorname{rank}\left(\mathcal{E}_{m, n}\right)=10$ are given in Table 3 ; the third number in it is the sum of two fourth powers in two different forms, and was first given in [12].

The search for candidates of high rank was done with Mathematica $^{\circledR}$, and the computations of the rank with mwrank.

ACKNOWLEDGEMENTS.

We thank Andrej Dujella for his insightful comments that resulted in an improvement of the paper. In particular, he pointed to us the final argument in the proof of Theorem 2.1. We also thank the Faculty of Science and 
TABlE 3. Curves of rank 10

\begin{tabular}{rrr}
\hline$m^{4}+n^{4}$ & $m$ & $n$ \\
\hline 6101085725427704897 & 24523 & 48946 \\
8353777080227411377 & 23637 & 53252 \\
173329443404113736737 & 15209 & 114732 \\
568859481611282619697 & 73239 & 152446 \\
715759918793283216257 & 124526 & 147653 \\
7288986688462077146017 & 67021 & 163162 \\
1195646546203028886017 & 132698 & 172507 \\
3314733655451433130177 & 118897 & 236244 \\
3358369470733089318817 & 109698 & 238093 \\
\hline
\end{tabular}

Technology of the University of the Basque Country for allowing us the use of one of the computer rooms, were computations were done on two weekends.

\section{REFERENCES}

[1] J. Aguirre, F. Castañeda and J. C. Peral, High rank elliptic curves with torsion group $\mathbb{Z} /(2 \mathbb{Z})$, Math. Comp. 73 (2004), 323-331

[2] J. Aguirre and J. C. Peral, Biquadrates and elliptic curves, preprint, http://arxiv. org/abs/1203.2576.

[3] W. P. Barth, K. Hulek, C. A. M. Peters and A. Van de Ven, Compact complex surfaces, Results in Mathematics and Related Areas. 3rd Series. A Series of Modern Surveys in Mathematics 4, Springer-Verlag, Berlin, 2004.

[4] G. Campbell, Finding elliptic curves and families of elliptic curves over $\mathbb{Q}$ of large rank, Thesis (Ph.D.)-Rutgers The State University of New Jersey - New Brunswick, ProQuest LLC, Ann Arbor, MI, 1999.

[5] J. E. Cremona, Algorithms for modular elliptic curves, Cambridge University Press, Cambridge, 1997.

[6] L. E. Dickson, History of the theory of numbers. Vol. II: Diophantine analysis, Chelsea Publishing Co., New York, 1966.

[7] A. Dujella, High rank elliptic curves with prescribed torsion, http://web.math.hr/ $\sim$ duje/tors/tors.html.

[8] S. Fermigier, Construction of high-rank elliptic curves over $\mathbb{Q}$ and $\mathbb{Q}(t)$ with nontrivial 2-torsion (extended abstract), Algorithmic number theory, Talence, 1996., Lecture Notes in Comput. Sci. 1122, Springer, Berlin, 1996, 115-120.

[9] Y. Fujita and N. Terai, On the rank of the elliptic curve $y^{2}=x^{3}-n x$, Int. J. Algebra 6 (2012), 885-901.

[10] R. K. Guy, Unsolved problems in number theory, Springer-Verlag, New York, 2004.

[11] G. H. Hardy and E. M. Wright, An introduction to the theory of numbers, Oxford University Press, Oxford, 2008.

[12] F. A. Izadi, F. Khoshnam and K. Nabardi, Sum of two biquadrates and elliptic curves of rank $\geq 4$, preprint, http://arxiv.org/abs/1202.5676

[13] S. Kihara, On the rank of the elliptic curve $y^{2}=x^{3}+k x$, Proc. Japan Acad. Ser. A Math. Sci. 74 (1998), 115-116

[14] S. Kihara, On the rank of the elliptic curve $y^{2}=x^{3}+k x$ II, Proc. Japan Acad. Ser. A Math. Sci. 80 (2004), 24-25. 
[15] Wolfram Research, Mathematica Edition: Version 8.0, Champaign, Illinois, 2010.

[16] R. Miranda, An overview of algebraic surfaces, Algebraic geometry, Ankara, 1995. Lecture Notes in Pure and Appl. Math. 193, Dekker, New York, 1997, 157-217,

[17] K. Nagao, On the rank of elliptic curve $y^{2}=x^{3}-k x$, Kobe J. Math., 11 (1994), 205-210.

[18] K. Ono and T. Ono, Quadratic forms and elliptic curves. III, Proc. Japan Acad. Ser. A Math. Sci. 72 (1996), 204-205.

[19] N. F. Rogers, Rank computations for the congruent number elliptic curves, Experiment. Math. 9 (2000), 591-594.

[20] T. Shioda, On the Mordell-Weil lattices, Comment. Math. Univ. St. Paul. 39 (1990), $211-240$.

[21] T. Shioda, Construction of elliptic curves with high rank via the invariants of the Weyl groups, J. Math. Soc. Japan 43 (1991), 673-719.

[22] J. H. Silverman, Advanced topics in the arithmetic of elliptic curves, Graduate Texts in Mathematics 151, Springer-Verlag, New York, 1994.

[23] J. Steggall, The Equation $x^{3}-u^{3}=y^{3}-v^{3}$ when $x, y, u, v$ are rational, Proc. Edinburgh Math. Soc. 34 (1915), 41-44.

[24] J. Wroblewski, Equal Sums of Powers-Tables, http://www.math.uni.wroc.pl/ jwr/ eslp/tables.htm

[25] A. J. Zajta, Solutions of the Diophantine equation $A^{4}+B^{4}=C^{4}+D^{4}$, Math. Comp. 41 (1983), 635-659.

J. Aguirre

Departamento de Matemáticas

Universidad del País Vasco UPV/EHU

Aptdo. 644, 48080 Bilbao

Spain

E-mail: julian.aguirre@ehu.es

J. C. Peral

Departamento de Matemáticas

Universidad del País Vasco UPV/EHU

Aptdo. 644, 48080 Bilbao

Spain

E-mail: juancarlos.peral@ehu.es

Received: 26.10.2012.

Revised: 30.11.2012. 